\title{
Second generation of composite fermions in the Hamiltonian theory
}

\author{
M. O. Goerbig ${ }^{1,2}$, P. Lederer ${ }^{2}$, and C. Morais Smith ${ }^{1}$ \\ ${ }^{1}$ Département de Physique, Université de Fribourg, Pérolles, CH-1700 Fribourg, Switzerland. \\ ${ }^{2}$ Laboratoire de Physique des Solides, Bat.510, UPS (associé au CNRS), F-91405 Orsay cedex, France.
}

\begin{abstract}
In the framework of a recently developed model of interacting composite fermions restricted to a single level, we calculate the activation gaps of a second generation of spin-polarized composite fermions. These composite particles consist each of a composite fermion of the first generation and a vortex-like excitation and may be responsible for the recently observed fractional quantum Hall states at unusual filling factors such as $\nu=4 / 11,5 / 13,5 / 17$, and $6 / 17$. Because the gaps of composite fermions of the second generation are found to be more than one order of magnitude smaller than those of the first generation, these states are less visible than the usual states observed at filling factors $\nu=p /(2 p s+1)$. Their stability is discussed in the context of a pseudopotential expansion of the composite-fermion interaction potential.
\end{abstract}

PACS numbers: 73.43.Cd, 71.10.Pm

\section{INTRODUCTION}

The fractional quantum Hall effect (FQHE) in the lowest Landau level (LL) at filling factors $\nu=p /(2 p s+1)$, with integral $p$ and $s$, may be understood as an integral quantum Hall effect (IQHE) in terms of composite fermions (CFs) $\stackrel{1.2}{=}$ Each CF consists of a bound state of one electron and a vortex-like excitation with vorticity $2 s$ and charge $c^{2}=2 p s /(2 p s+1)$, in units of the electron charge. The CFs experience a reduced coupling $(e B)^{*}=$ $e B /(2 p s+1)$ to the external magnetic field $B$ and thus populate CF-LLs with a CF filling factor $\nu^{*}=n_{e l} / n_{B^{*}}$. Here, $n_{e l}$ is the electronic density, and $n_{B^{*}}=1 / 2 \pi l_{B}^{* 2}$ is the density of states of each CF-LL in terms of the CF magnetic length $l_{B}^{*}=\sqrt{\hbar /(e B)^{*}}$. The CF and electronic filling factors are related by $\nu=\nu^{*} /\left(2 s \nu^{*}+1\right)$. The FQHE arises when $\nu^{*}=p$ : because of the CF-LL separation, which is on the order of the characteristic Coulomb energy $e^{2} / \epsilon l_{B}$, a finite energy is required to promote a particle to an upper CF-LL if the filling is changed. These particles become localized by residual impurities and therefore do not contribute to the electrical transport, giving rise to the observed plateaus in the Hall resistance.

Experiments by Pan et al. have revealed a new class of FQHE states at filling factors such as $\nu=4 / 11,6 / 17, \ldots$, of which at least the $4 / 11$ state is found to be spinpolarized ${ }^{\underline{3}}$ The existence of such states had been conjectured by Mani and v. Klitzing based on the fractal structure and self-similarity of the Hall resistance curve $\underline{4}$ It appears natural to interpret these states, found at CFfilling factors $\nu^{*}=1+\tilde{p} /(2 \tilde{s} \tilde{p}+1)$, in terms of an IQHE of a second generation of CFs $\left(\mathrm{C}^{2} \mathrm{Fs}\right)$; whereas the CFs in the lowest CF-LL $(p=0)$ remain inert, those in the partially filled $p=1$ level bind to some vortex-like excitation carrying $2 \tilde{s}$ additional flux quanta. This picture is reminiscent of the hierarchy scheme proposed by Haldane and Halperin, according to which Laughlin quasiparticles ${ }^{5}$ might in principle form an incompressible liquid state if the interaction potential between these quasiparticles is sufficiently short-range ${ }^{6.7}$ Even though the new states may be classified within this scheme, theoretical studies seem to indicate that fully spin-polarized $\mathrm{C}^{2} \mathrm{Fs}$ and even higher generations of CFs would not be stable. , $9,10,11^{-1}$ Exact-diagonalization studies for the $4 / 11$ state show a gapped ground state only for 12 electrons, which is at present the maximum electron number for such numerical investigations $\stackrel{12}{2}$ On the other hand, a numerical diagonalization with up to 24 particles can be performed in the $\mathrm{CF}$ basis with a truncated Hilbert space ${ }^{10.11}$ Within the latter formalism, a ground state with features of a FQHE state emerges for $N=12$ and 20 particles, but not for $N=8,16$, and $241^{10}$ The extrapolation to the thermodynamic limit is therefore ambiguous. Lopez and Fradkin investigated the topological stability of a FQHE state at $\nu=4 / 11$ in a Chern-Simons field-theory approach ${ }^{13} \mathrm{In}$ contrast to prior stability investigations by Haldane, $\stackrel{14}{=}$ a spin-polarized state is found to be topologically stable, if interpreted in terms of $\mathrm{C}^{2} \mathrm{Fs}$.

Recently, we have derived a model of interacting $\mathrm{CF}^{15}$ in the framework of the Hamiltonian theory proposed by Murthy and Shankar ${ }^{16}$ The involved transformations provide a mathematical basis for the self-similarity of the FQHE $\stackrel{15}{\underline{15}}$ Here, we construct a $\mathrm{C}^{2} \mathrm{~F}$ representation of this model, in analogy with the $\mathrm{CF}$ representation in the Hamiltonian theory, by the restriction of the dynamics to the first excited CF-LL $p=1$. We suppose the stability of fully spin-polarized $\mathrm{C}^{2} \mathrm{~F}$ states and calculate their activation gaps as a function of the finite width of the two-dimensional electron system. In contrast to prior numerical analyses in the wave-function approach, the calculations are performed directly in the thermodynamic limit. Inter-CF-LL excitations are taken into account and give rise to a screened effective interaction. The dielectric function, which is calculated in the randomphase approximation (RPA), is similar to the electronic case discussed by Aleiner and Glazman $\underline{17}$

The structure of the paper is the following: in Sec. II we present the model of interacting CFs and evaluate their effective interaction potential. The dielectric function due to inter-CF-LL excitations is derived in Sec. III. In Sec. IV we construct the $\mathrm{C}^{2} \mathrm{~F}$ basis and calculate the 
activation gaps of the $\mathrm{C}^{2} \mathrm{~F}$ states as a function of the finite width of the electron system. The resulting activation gap for $\nu=4 / 11$ is compared to the experimental estimate. The stability of $\mathrm{C}^{2} \mathrm{~F}$ states is discussed in terms of a pseudopotential expansion of the CF interaction potential in Sec. V. A brief summary may be found in Sec. VI.

\section{MODEL}

We adopt a model of spin-polarized particles, the dynamics of which are restricted to the lowest electronic LL. The low-energy degrees of freedom are given by the Hamiltonian

$$
\hat{H}=\frac{1}{2} \sum_{\mathbf{q}} v_{0}(q) \bar{\rho}(-\mathbf{q}) \bar{\rho}(\mathbf{q})
$$

where $v_{0}(q)=\left(2 \pi e^{2} / \epsilon q\right) \exp \left(-q^{2} l_{B}^{2} / 2\right)$, with $l_{B}=$ $\sqrt{\hbar / e B}$. Instead of representing the projected density operators $\bar{\rho}(\mathbf{q})$ in the usual electron basis, an alternative representation in terms of CFs is chosen in the Hamiltonian theory of the FQHE: a "preferred" combination of the projected density and the density $\bar{\chi}(\mathbf{q})$ of the vortexlike object is introduced, $\bar{\rho}_{C F}(\mathbf{q})=\bar{\rho}(\mathbf{q})-c^{2} \bar{\chi}(\mathbf{q}) 16$ This preferred combination plays the role of the CF density operator and may be expressed as

$$
\begin{aligned}
\bar{\rho}_{C F}(\mathbf{q})= & \sum_{n, n^{\prime} ; m, m^{\prime}}\left\langle m\left|e^{-i \mathbf{q} \cdot \mathbf{R}_{C F}}\right| m^{\prime}\right\rangle \\
& \times\left\langle n\left|\bar{\rho}^{p}(\mathbf{q})\right| n^{\prime}\right\rangle c_{n, m}^{\dagger} c_{n^{\prime}, m^{\prime}}
\end{aligned}
$$

where $c_{n, m}^{\dagger}$ creates a CF in the $n$-th CF-LL with the guiding-center quantum number $m$. The matrix elements are $\left(\right.$ for $m \geq m^{\prime}$ )

$$
\begin{aligned}
\left\langle m\left|e^{-i \mathbf{q} \cdot \mathbf{R}_{C F}}\right| m^{\prime}\right\rangle= & \sqrt{\frac{m^{\prime} !}{m !}}\left(\frac{-i\left(q_{x}+i q_{y}\right) l_{B}^{*}}{\sqrt{2}}\right)^{m-m^{\prime}} \\
& L_{m^{\prime}}^{m-m^{\prime}}\left(\frac{q^{2} l_{B}^{* 2}}{2}\right) e^{-q^{2} l_{B}^{* 2} / 4}
\end{aligned}
$$

and (for $n \geq n^{\prime}$ )

$$
\begin{aligned}
\left\langle n\left|\bar{\rho}^{p}(\mathbf{q})\right| n^{\prime}\right\rangle= & \sqrt{\frac{n^{\prime} !}{n !}}\left(\frac{-i\left(q_{x}-i q_{y}\right) l_{B}^{*} c}{\sqrt{2}}\right)^{n-n^{\prime}} e^{-q^{2} l_{B}^{* 2} c^{2} / 4} \\
& \times\left[L_{n^{\prime}}^{n-n^{\prime}}\left(\frac{q^{2} l_{B}^{* 2} c^{2}}{2}\right)\right. \\
& \left.-c^{2\left(1-n+n^{\prime}\right)} e^{-q^{2} l_{B}^{2} / 2 c^{2}} L_{n^{\prime}}^{n-n^{\prime}}\left(\frac{q^{2} l_{B}^{* 2}}{2 c^{2}}\right)\right]
\end{aligned}
$$

with the associated Laguerre polynomials $L_{n}^{m}(x)$ and the CF magnetic length $l_{B}^{*}=l_{B} / \sqrt{1-c^{2}}$.

In order to describe the low energy degrees of freedom in the experimentally relevant range of $\mathrm{CF}$ fillings $1<$ $\nu^{*}<2$, we restrict $\bar{\rho}_{C F}(\mathbf{q})$ to the CF-LL $p=1$ in the same manner as for electrons. The restricted CF-density operator is $\left\langle\bar{\rho}_{C F}(\mathbf{q})\right\rangle_{p=1}=\left\langle 1\left|\bar{\rho}^{p}(\mathbf{q})\right| 1\right\rangle \overline{\bar{\rho}}(\mathbf{q})$, with

$$
\overline{\bar{\rho}}(\mathbf{q})=\sum_{m, m^{\prime}}\left\langle m\left|e^{-i \mathbf{q} \cdot \mathbf{R}_{C F}}\right| m^{\prime}\right\rangle c_{n=1, m}^{\dagger} c_{n=1, m^{\prime}} .
$$

The model Hamiltonian of restricted CFs therefore becomes 15

$$
\tilde{H}(s)=\frac{1}{2} \sum_{\mathbf{q}} \tilde{v}(q) \overline{\bar{\rho}}(-\mathbf{q}) \overline{\bar{\rho}}(\mathbf{q}),
$$

where the CF form factor

$$
\begin{aligned}
F_{C F}(q) \equiv\left\langle 1\left|\bar{\rho}^{p}(\mathbf{q})\right| 1\right\rangle & =e^{-q^{2} l_{B}^{* 2} c^{2} / 4}\left[L_{1}\left(\frac{q^{2} l_{B}^{* 2} c^{2}}{2}\right)\right. \\
& \left.-c^{2} e^{-q^{2} l_{B}^{2} / 2 c^{2}} L_{1}\left(\frac{q^{2} l_{B}^{* 2}}{2 c^{2}}\right)\right]
\end{aligned}
$$

has been absorbed into an effective CF interaction potential

$$
\tilde{v}(q)=v_{0}(q) \frac{\left[F_{C F}(q)\right]^{2}}{\epsilon(q)} .
$$

Note that the derivation of this CF model is not limited to $p=1$, but may also be applied for higher CF-LLs. In this case the CF form factor depends on $p, F_{C F}^{p}(q)=$ $\left\langle p\left|\bar{\rho}^{p}(\mathbf{q})\right| p\right\rangle$. The projected CF-density operators satisfy the same commutation relations

$$
[\overline{\bar{\rho}}(\mathbf{q}), \overline{\bar{\rho}}(\mathbf{k})]=2 i \sin \left(\frac{(\mathbf{q} \times \mathbf{k})_{z} l_{B}^{* 2}}{2}\right) \overline{\bar{\rho}}(\mathbf{q}+\mathbf{k})
$$

as the projected density operators in the model of electrons restricted to the lowest LL if one replaces the electronic by the CF magnetic length. ${ }^{16.18}$ This and the fact that the Hamiltonian of interacting CFs (4) has the same structure as the original electronic one ${ }^{15}$ allows us treat the model with the same theoretical tools which have been used for the understanding of the FQHE at $\nu=p /(2 p s+1)$. One would simply need to replace the electronic by the $\mathrm{CF}$ interaction potential, $v_{0}(q) \rightarrow \tilde{v}(q)$, and take into account the renormalization of the magnetic length, $l_{B} \rightarrow l_{B}^{*}$.

\section{DIELECTRIC FUNCTION}

In contrast to the electronic case, inter-CF-LL excitations have to be considered explicitly in a dielectric function $\epsilon(q)$, which modifies the CF interaction potential, because the residual $\mathrm{CF}$ interactions are intrinsically on the same order of magnitude as the CF-LL separation both energy scales are given by the Coulomb interaction $e^{2} / \epsilon l_{B}$.

For the calculation of the dielectric function, we investigate inter-CF-LL excitations in the original Hamiltonian (11) in the CF basis. Its solution in the Hartree-Fock approximation yields the "free" CF Hamiltonian

$$
\hat{H}_{C F}^{0}=\sum_{n, m} \varepsilon_{n} c_{n, m}^{\dagger} c_{n, m} .
$$


It can be shown that the CF-LLs are approximately linear in $n, \epsilon_{n} \simeq n \omega_{C}^{*}$, where $\omega_{C}^{*}$ is the energy of a quasiparticle excitation in units of $\hbar \equiv 1$, and we have omitted an unimportant constant. The corrections beyond the Hartree-Fock approximation are given by the "interaction" Hamiltonian

$$
\begin{aligned}
\hat{H}_{C F}^{i n t}= & \frac{1}{2} \sum_{\nu_{1}, \ldots, \nu_{4}} v_{\nu_{1}, \ldots, \nu_{4}}\left[c_{\nu_{1}}^{\dagger} c_{\nu_{2}}^{\dagger} c_{\nu_{4}} c_{\nu_{3}}\right. \\
& -\left\langle c_{\nu_{1}}^{\dagger} c_{\nu_{3}}\right\rangle c_{\nu_{2}}^{\dagger} c_{\nu_{4}}-\left\langle c_{\nu_{2}}^{\dagger} c_{\nu_{4}}\right\rangle c_{\nu_{1}}^{\dagger} c_{\nu_{3}} \\
& \left.+\left\langle c_{\nu_{1}}^{\dagger} c_{\nu_{4}}\right\rangle c_{\nu_{2}}^{\dagger} c_{\nu_{3}}+\left\langle c_{\nu_{2}}^{\dagger} c_{\nu_{3}}\right\rangle c_{\nu_{1}}^{\dagger} c_{\nu_{4}}\right],
\end{aligned}
$$

with $\nu_{i}=\left(n_{i}, m_{i}\right)$ and the interaction vertex

$$
\begin{aligned}
v_{\nu_{1}, \ldots, \nu_{4}}= & \sum_{\mathbf{q}} v_{0}(q)\left\langle n_{1}\left|\bar{\rho}^{p}(-\mathbf{q})\right| n_{3}\right\rangle\left\langle n_{2}\left|\bar{\rho}^{p}(\mathbf{q})\right| n_{4}\right\rangle \\
& \left\langle m_{1}\left|e^{i \mathbf{q} \cdot \mathbf{R}_{C F}}\right| m_{3}\right\rangle\left\langle m_{2}\left|e^{-i \mathbf{q} \cdot \mathbf{R}_{C F}}\right| m_{4}\right\rangle .
\end{aligned}
$$

The special form of the interaction Hamiltonian (7) leads to the omission of all diagrams containing the equaltime contractions $\left\langle\mathcal{T} c_{n, m}(\tau) c_{n, m}^{\dagger}(\tau)\right\rangle$ in a diagrammatic perturbation expansion, where $\mathcal{T}$ denotes time-ordering. The averages are taken over the Hartree-Fock ground state characterized by $\left\langle c_{n, m}^{\dagger} c_{n^{\prime}, m^{\prime}}\right\rangle=\delta_{n, n^{\prime}} \delta_{m, m^{\prime}} \Theta(p-1-$ $n)$, where $\Theta(x)$ is the step function with $\Theta(x)=0$ for $x<$ 0 and $\Theta(x)=1$ for $x \geq 0$. The dielectric function may be calculated in the RPA, $\epsilon_{R P A}(\mathbf{q}, \omega)=1-v_{0}(q) D(\mathbf{q}, \omega)$, where

$$
\begin{aligned}
D(\mathbf{q}, \omega) & =-i \int d t e^{i \omega t}\left\langle\mathcal{T} \bar{\rho}_{C F}(\mathbf{q}, t) \bar{\rho}_{C F}(-\mathbf{q}, t=0)\right\rangle \\
& =2 \sum_{n>0} \mathcal{F}_{n}^{p}(q) \frac{n \omega_{C}^{*}}{\omega^{2}-n^{2} \omega_{C}^{* 2}}
\end{aligned}
$$

is the density-density Green's function with

$$
\mathcal{F}_{n}^{p}(q)=\sum_{n^{\prime}=p-n}^{p-1}\left|\left\langle n^{\prime}+n\left|\bar{\rho}^{p}(\mathbf{q})\right| n^{\prime}\right\rangle\right|^{2}
$$

For lower $p$, the static dielectric function in the limit $\omega \rightarrow 0$ is a finite sum over Laguerre polynomials, which may be directly calculated with the help of the matrix elements (3). In the case of $p=1$ one finds

$$
\begin{aligned}
\epsilon_{R P A}^{(p=1)}(q)= & 1+\frac{v_{0}(q)\left(q l_{B}^{*} c\right)^{2}}{\omega_{C}^{*}}\left[L_{1}\left(\frac{q^{2} l_{B}^{* 2} c^{2}}{2}\right)\right. \\
& \left.-e^{-q^{2} l_{B}^{2} / 2 c^{2}} L_{1}\left(\frac{q^{2} l_{B}^{* 2}}{2 c^{2}}\right)\right]^{2} e^{-q^{2} l_{B}^{* 2} c^{2} / 2} .
\end{aligned}
$$

For larger values of $p$, one may use the asymptotic form of the amplitudes (8)

$$
\mathcal{F}_{n}^{p}(q) \simeq n\left[J_{n}\left(q l_{B}^{*} c \sqrt{2 p+1}\right)-c^{2} J_{n}\left(\frac{q l_{B}^{*}}{c} \sqrt{2 p+1}\right)\right]^{2},
$$

in terms of the Bessel functions $J_{n}(x)$. This yields the static dielectric function

$$
\epsilon_{R P A}^{p}(q) \simeq 1+\frac{v_{0}(q)}{\omega_{C}^{*}}\left\{1+c^{4}-2 c^{2} J_{0}\left[q R_{C}^{*}\left(c-\frac{1}{c}\right)\right]\right.
$$

$$
\left.-\left[J_{0}\left(q R_{C}^{*} c\right)-c^{2} J_{0}\left(\frac{q R_{C}^{*}}{c}\right)\right]^{2}\right\}
$$

with the CF cyclotron radius $R_{C}^{*}=l_{B}^{*} \sqrt{2 p+1}$. This is similar to the sreening of the effective electron-electron interaction in higher electronic LLs 17 Note that the RPA is more problematic in the $\mathrm{CF}$ than in the electronic case because $\left(e^{2} / \epsilon l_{B}\right) / \omega_{C}^{*}$ is not a physically small parameter. Therefore, other diagrams may also play a role in the dielectric function.

\section{C ${ }^{2}$ F BASIS AND ACTIVATION GAPS}

In order to obtain the $\mathrm{C}^{2} \mathrm{~F}$ representation of the model, we proceed in the same manner as for the construction of the CF representation ${ }^{16} \mathrm{~A}$ new preferred combination is introduced, $\bar{\rho}_{C^{2} F}(\mathbf{q})=\overline{\bar{\rho}}(\mathbf{q})-\tilde{c}^{2} \overline{\bar{\chi}}(\mathbf{q})$, where $\overline{\bar{\chi}}(\mathbf{q})$ describes a vortex-like excitation of the $\mathrm{CF}$ liquid with vorticity $2 \tilde{s}$ and charge $\tilde{c}^{2}=2 \tilde{p} \tilde{s} /(2 \tilde{p} \tilde{s}+1)$ in units of the CF charge $e^{*}=1-c^{2}$. This density operator becomes in the $\mathrm{C}^{2} \mathrm{~F}$ basis

$$
\begin{aligned}
\bar{\rho}_{C^{2} F}(\mathbf{q})= & \sum_{n, n^{\prime} ; m, m^{\prime}}\langle m| e^{-i \mathbf{q} \cdot \mathbf{R}_{C^{2} F}\left|m^{\prime}\right\rangle} \\
& \times\left\langle n\left|\overline{\bar{\rho}}^{p}(\mathbf{q})\right| n^{\prime}\right\rangle \tilde{c}_{n, m}^{\dagger} \tilde{c}_{n^{\prime}, m^{\prime}}
\end{aligned}
$$

where $\tilde{c}_{n, m}^{\dagger}$ creates a $\mathrm{C}^{2} \mathrm{~F}$ in the $\mathrm{C}^{2} \mathrm{~F}$-LL $n$ in the guiding-center state $m$. The degeneracy of each $\mathrm{C}^{2} \mathrm{~F}$ $\mathrm{LL}$ is now $n_{\tilde{B}}=1 / 2 \pi \tilde{l}^{2}$, where $\tilde{l}=l_{B}^{*} / \sqrt{1-\tilde{c}^{2}}=$ $l_{B} / \sqrt{\left(1-c^{2}\right)\left(1-\tilde{c}^{2}\right)}$ is the $\mathrm{C}^{2} \mathrm{~F}$ magnetic length. The matrix elements are given by Eqs. (2) and (3) if one replaces $l_{B}^{*} \rightarrow \tilde{l}$ and $c \rightarrow \tilde{c}$.

The activation gap for $\mathrm{C}^{2} \mathrm{Fs}$ is calculated in the same manner as for CFs; 16 it is the sum of the energies of a quasiparticle and a quasihole excitation $\Delta^{a}(s ; \tilde{s}, \tilde{p})=$ $\Delta^{q p}(s ; \tilde{s}, \tilde{p})+\Delta^{q h}(s ; \tilde{s}, \tilde{p})$, with

$$
\Delta^{q p}(s ; \tilde{s}, \tilde{p})=\left\langle\tilde{c}_{\tilde{p}, m} \tilde{H}(s) \tilde{c}_{\tilde{p}, m}^{\dagger}\right\rangle-\langle\tilde{H}\rangle
$$

and

$$
\Delta^{q h}(s ; \tilde{s}, \tilde{p})=\left\langle\tilde{c}_{\tilde{p}-1, m}^{\dagger} \tilde{H}(s) \tilde{c}_{\tilde{p}-1, m}\right\rangle-\langle\tilde{H}\rangle .
$$

With the help of Wick contractions, one averages over the $\mathrm{C}^{2} \mathrm{~F}$ ground state, which consists of $\tilde{p}$ completely filled $\mathrm{C}^{2} \mathrm{~F}$-LLs and may thus be characterized by $\left\langle\tilde{c}_{n, m}^{\dagger} \tilde{c}_{n^{\prime}, m^{\prime}}\right\rangle=\delta_{n, n^{\prime}} \delta_{m, m^{\prime}} \Theta(\tilde{p}-1-n)$. This yields the activation gaps

$$
\begin{array}{r}
\Delta^{a}(s ; \tilde{s}, \tilde{p})=\frac{1}{2} \sum_{\mathbf{q}} \tilde{v}(q)\left[\left\langle\tilde{p}\left|\overline{\bar{\rho}}^{p}(-\mathbf{q}) \overline{\bar{\rho}}^{p}(\mathbf{q})\right| \tilde{p}\right\rangle\right. \\
\left.-\left\langle\tilde{p}-1\left|\overline{\bar{\rho}}^{p}(-\mathbf{q}) \overline{\bar{\rho}}^{p}(\mathbf{q})\right| \tilde{p}-1\right\rangle\right] \\
-\sum_{\mathbf{q}} \tilde{v}(q) \sum_{n=0}^{\tilde{p}-1}\left[\left|\left\langle\tilde{p}\left|\overline{\bar{\rho}}^{p}(\mathbf{q})\right| n\right\rangle\right|^{2}-\left|\left\langle\tilde{p}-1\left|\overline{\bar{\rho}}^{p}(\mathbf{q})\right| n\right\rangle\right|^{2}\right],
\end{array}
$$

in analogy with the case of CFs, 16 
In units of $e^{2} / \epsilon l_{B}$ and for characteristic quasiparticle energies $\omega_{C}^{*}$ found in the litterature,, 16.19 the activation gaps of some chosen states with $s=1$ and $p=1$ (with $\left.\omega_{C}^{*}=0.1\right)$ read

\begin{tabular}{|c||c|c|}
\hline$\tilde{s}=1$ & $\tilde{p}=1$ & $\tilde{p}=2$ \\
\hline$\nu^{*}$ & $1+1 / 3$ & $1+2 / 5$ \\
\hline$\nu$ & $4 / 11$ & $7 / 19$ \\
\hline \hline$\Delta^{a}$ & 0.0067 & 0.0027 \\
\hline
\end{tabular}

\begin{tabular}{|c||c|c|}
\hline$\tilde{s}=2$ & $\tilde{p}=1$ & $\tilde{p}=2$ \\
\hline$\nu^{*}$ & $1+1 / 5$ & $1+2 / 9$ \\
\hline$\nu$ & $6 / 17$ & $11 / 31$ \\
\hline \hline$\Delta^{a}$ & 0.0031 & 0.0013 \\
\hline
\end{tabular}

whereas for states with $s=2$ and $p=1$ (with $\omega_{C}^{*}=0.03$ ), one obtains

\begin{tabular}{|c||c|c|}
\hline$\tilde{s}=1$ & $\tilde{p}=1$ & $\tilde{p}=2$ \\
\hline$\nu^{*}$ & $1+1 / 3$ & $1+2 / 5$ \\
\hline$\nu$ & $4 / 19$ & $7 / 33$ \\
\hline \hline$\Delta^{a}$ & 0.0016 & 0.00070 \\
\hline
\end{tabular}

\begin{tabular}{|c||c|c|}
\hline$\tilde{s}=2$ & $\tilde{p}=1$ & $\tilde{p}=2$ \\
\hline$\nu^{*}$ & $1+1 / 5$ & $1+2 / 9$ \\
\hline$\nu$ & $6 / 29$ & $11 / 53$ \\
\hline \hline$\Delta^{a}$ & 0.00082 & 0.00035 \\
\hline
\end{tabular}

Note that the $\mathrm{C}^{2} \mathrm{~F}$ states at $\nu^{*}=1+\tilde{p} /(2 \tilde{p} \tilde{s}+1)$ and $\nu^{*}=$ $2-\tilde{p} /(2 \tilde{p} \tilde{s}+1)$ are related by the particle-hole symmetry in the same manner as the CF states at $\nu=p /(2 p s+1)$ and $\nu=1-p /(2 p s+1)$. The $4 / 11$ state is therefore equivalent to the states at $\nu=5 / 13,7 / 11$, and $8 / 13$.

Finite width effects may be included by replacing the original interaction potential $v_{0}(q) \rightarrow v_{0}(q) f(\lambda, q)$, where $f(\lambda, q)=\exp \left(\lambda^{2} q^{2}\right)[1-\operatorname{Erf}(\lambda q)]$ is given in terms of the error function $\operatorname{Erf}(x)$. This expression has been obtained under the assumption that the confining potential in the $z$-direction (with a characteristic width $\lambda$ ) be quadratic. ${ }^{16.19}$ The results are shown in Fig.1 for different filling factors. As expected from the FQHE of electrons, the activation gap is reduced for samples with larger $\lambda^{19}$

The only activation gap of this series which was inferred from experiments is the one at $\nu=4 / 11$, which is on the order of $50 \mathrm{mK}$ for a sample width of $\sim 500 \AA .3$ This corresponds to a width parameter $\lambda \simeq 6.3 l_{B}$ at $B=10 \mathrm{~T}$. The activation gap obtained theoretically for this width is $\Delta^{a}(s=1 ; \tilde{s}=1, \tilde{p}=1) \simeq 0.0025 e^{2} / \epsilon l_{B}$, which is on the order of $400 \mathrm{mK}$ and thus one order of magnitude larger than the experimental activation gap and almost three times larger than the value calculated by Chang et al. for a partially spin-polarized state. ${ }^{11}$ Two effects may account for this discrepancy. First, it is known that the Hamiltonian theory for the conventional FQHE overestimates the activation gap by a factor of $\sim 1.5$ in comparison with numerical studies even if the agreement becomes better for wider samples. ${ }^{16}$ This effect is expected to be also present in the $\mathrm{C}^{2} \mathrm{~F}$ formalism. A second and more likely origin of the discrepancy between the theoretical and experimental results is the fact that the theory does not account for impurity effects. They lead to a gap reduction, which may be on the same order of magnitude as the activation gap in the absence of impurities. ${ }^{20}$ Impurities affect these sensitive $\mathrm{C}^{2} \mathrm{~F}$ states more than their corresponding CF states because the activation gap of the latter is more than one order of magnitude larger.
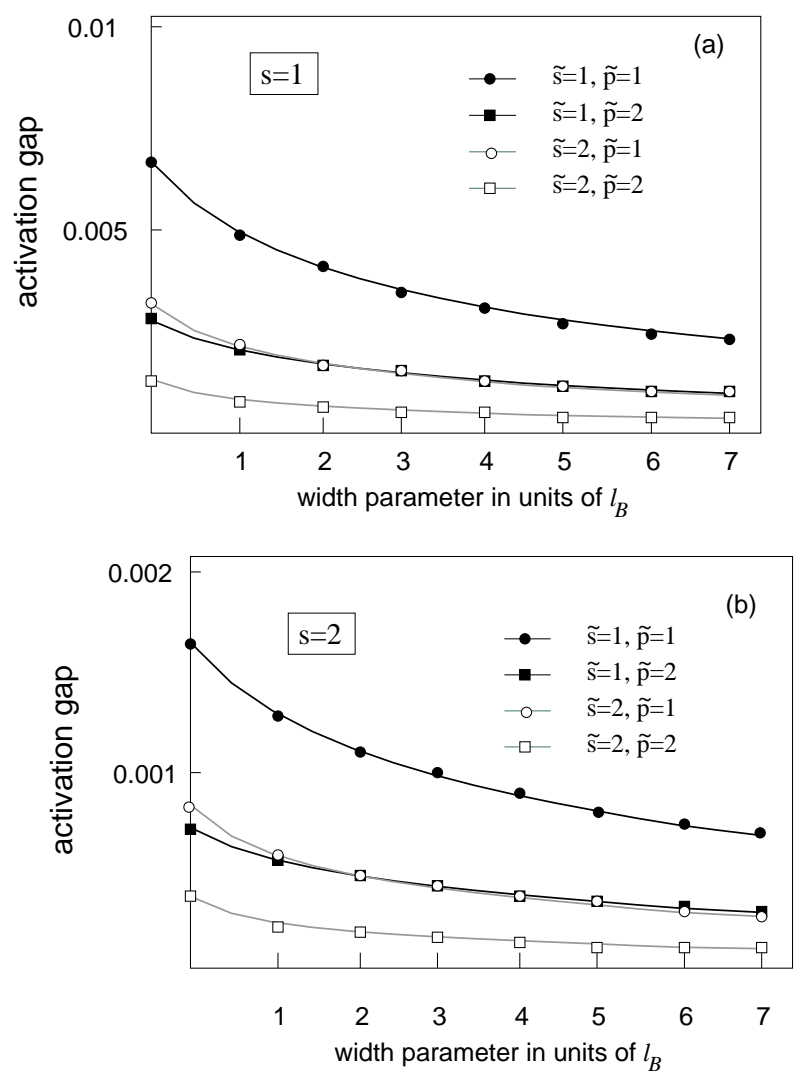

FIG. 1: Activation gaps as a function of width parameter for different fillings. (a) $s=1: \nu=4 / 11$ (black circles), $\nu=6 / 17$ (white circles), $\nu=7 / 19$ (black squares), and $\nu=11 / 31$ (white squares); (b) $s=2: \nu=4 / 19$ (black circles), $\nu=6 / 29$ (white circles), $\nu=7 / 33$ (black squares), and $\nu=11 / 53$ (white squares).

\section{CRITERION FOR THE STABILITY OF $\mathrm{C}^{2}$ FS}

Finally, we comment on the stability of $\mathrm{C}^{2} \mathrm{Fs}$. In order to conclude about their existence in nature, one would in principle have to compare the energy of the $\mathrm{C}^{2} \mathrm{~F}$ liquid with possibly competing phases, e.g. solid phases of CFs in $p=1,21$ However, insight into their stability may be obtained from an expansion of the CF interaction potential (5) in Haldane's pseudopotentials, ${ }^{6} V_{m}^{C F}=$ $\sum_{\mathbf{q}} \tilde{v}(q) L_{m}\left(q^{2} l_{B}^{2 *}\right) \exp \left(-q^{2} l_{B}^{2 *} / 2\right)$, with odd $m$. The results for $p=1$ and different $s$, i.e. for the CF interaction in the filling-factor range $1 /(2 s+1)<\nu<2 /(4 s+1)$, are shown in Fig.2 In contrast to the electronic case, one observes a non-monotonic behavior for the unscreened $\mathrm{CF}$ interaction (black lines); the largest value is found for $m=1$, but there is a large suppression of the component $V_{3}^{C F}$, which is smaller than $V_{5}^{C F}$ and even becomes negative for larger $s$. A similar result has been obtained in previous studies in the wave-function approach. ${ }^{8,21}$ The non-monotonic behavior is likely to be due to the dipolelike internal structure of the CF 16.22 .23 An attractive dipole-dipole interaction is thus superimposed on the repulsive Coulomb repulsion. This stabilizes the $\mathrm{C}^{2} \mathrm{~F}$ states 


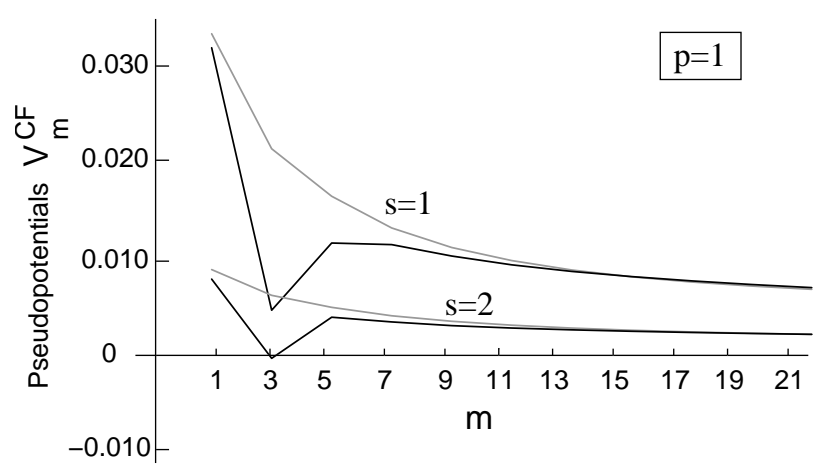

FIG. 2: Haldane's pseudopotentials for CF interaction both without screening (black line) and with screening (gray line); the lines are a guide to the eyes.

at $\nu^{*}=1+1 / 3$, such as the $4 / 11$ state, which have their largest weight in the $m=1$ angular-momentum component, but may destabilize states at $\nu^{*}=1+1 / 5$, e.g. the one at $\nu=6 / 17$. If one takes into account the screening, given by the dielectric function (9), the component $V_{3}^{C F}$ increases, and the potential becomes more Coulomb-like (gray lines). The $4 / 11$ state is found to remain stable in the presence of screening because for $s=1$ the ratio $V_{1}^{C F} / V_{3}^{C F}=1.56$ remains sufficiently high, i.e. the potential is sufficiently short-range. A first-order phase transition to a different state is expected to appear for a ratio $V_{1} / V_{3} \lesssim 1.22^{24}$

\section{CONCLUSIONS}

In conclusion, we have interpreted the experimentally observed $\mathrm{FQHE}^{3}$ at $\nu=4 / 11$ as an IQHE of a second generation of $\mathrm{CFs}\left(\mathrm{C}^{2} \mathrm{Fs}\right)$ in the framework of a recently developed model of interacting $\mathrm{CFs}, \frac{15}{15}$ which is based on the Hamiltonian theory of the FQHE 16 After restriction of the dynamics to the CF-LL $p=1$, the effective Hamiltonian for $\mathrm{C}^{2} \mathrm{Fs}$ becomes similar to the $\mathrm{CF}$ Hamiltonian in the lowest electronic LL. The formalism is generic and may be applied for other CF-LLs, $p>1$, as well as for higher generations of CFs $\underline{\underline{15}}$ Screening effects due to inter-CF-LL excitations, which in contrast to their electronic counterpart cannot be neglected even in the large- $B$ limit, are included in a dielectric function, calculated in the RPA. Both screening and the finite width of the two-dimensional electron gas give rise to a modification of the effective interaction potential and lead to a reduction of the $\mathrm{C}^{2} \mathrm{~F}$ activation gaps, which are about one order of magnitude smaller than those for CFs. The theoretical gap at $\nu=4 / 11$ is, however, substantially larger than the gap inferred from the experiment ${ }^{3}$ This discrepancy is likely to be due to residual impurities in the sample, which have been omitted in the theoretical model and which are known to produce a further reduction of the activation gaps ${ }^{20}$ Whereas the stability of a fully polarized 4/11 state remains controversial, 8.9.10,13,14 a pseudopotential expansion of the CF interaction potential hints to a stable $4 / 11$ state if interpreted in terms of $\mathrm{C}^{2} \mathrm{Fs}$. The definitive answer to this question, however, requires detailed energy calculations involving all possible competing states, namely CF Wigner crystal, CF bubble, and $\mathrm{C}^{2} \mathrm{~F}$ liquid phases.

We acknowledge fruitful discussions with R. Morf. This work was supported by the Swiss National Foundation for Scientific Research under Grant No. 62062868.00 .
1 J. K. Jain, Phys. Rev. Lett. 63, 199 (1989); Phys. Rev. B 41, 7653 (1990).

2 S. Das Sarma and A. Pinczuk, eds., Perspectives in Quantum Hall Effects, Wiley, New York (1997).

${ }^{3}$ W. Pan, H. L. Stormer, D. C. Tsui, L. N. Pfeiffer, K. W. Baldwin, and K. W. West, Phys. Rev. Lett. 90, 016801 (2003).

4 R. G. Mani and K. v. Klitzing, Z. Phys. B 100, 635 (1996).

${ }^{5}$ R. B. Laughlin, Phys. Rev. Lett. 50, 1395 (1983).

${ }^{6}$ F. D. Haldane, Phys. Rev. Lett. 51, 605 (1983).

7 B. I. Halperin, Phys. Rev. Lett. 52, 1583 (1984).

${ }^{8}$ P. Béran and R. Morf, Phys. Rev. B 43, 12654. (1991).

9 A. Wójs and J. J. Quinn, Phys. Rev. B 61, 2846 (2000).

10 S. S. Mandal and J. K. Jain, Phys. Rev. B 66, 155302 (2002).

11 C.-C. Chang, S. S. Mandal, and J. K. Jain, Phys. Rev. B 67, 121305 (2003).

12 R. Morf, private communication.

13 A. Lopez and E. Fradkin, cond-mat/0310128

14 F. D. Haldane, Phys. Rev. Lett. 74, 2090 (1995).
15 M. O. Goerbig, P. Lederer, and C. Morais Smith, cond-mat/0401340

16 G. Murthy and R. Shankar, Rev. Mod. Phys. 75, 1101 (2003); R. Shankar, Phys. Rev. B 63, 085322 (2001).

17 I. L. Aleiner and L. I. Glazman, Phys. Rev. B 52, 11296 (1995).

18 S. M. Girvin, A. H. MacDonald, and P. M. Platzman, Phys. Rev. B 33, 2481 (1986).

19 R. Morf, N. d'Ambrumenil, and S. Das Sarma, Phys. Rev. B 66, 075408 (2002).

${ }^{20}$ R. Morf and N. d'Ambrumenil, Phys. Rev. B 68, 113309 (2003).

21 S.-Y. Lee, V. W. Scarola, and J. K. Jain, Phys. Rev. Lett. 87, 256803 (2001); Phys. Rev. B 66, 085336 (2002).

${ }^{22}$ N. Read, Semi. Sci. Tech. 9, 1859 (1994).

23 V. Pasquier and F. D. Haldane, Nucl. Phys. B 516, 719 (1998).

24 D. Yoshioka, The Quantum Hall Effect, Springer, Berlin (2002). 

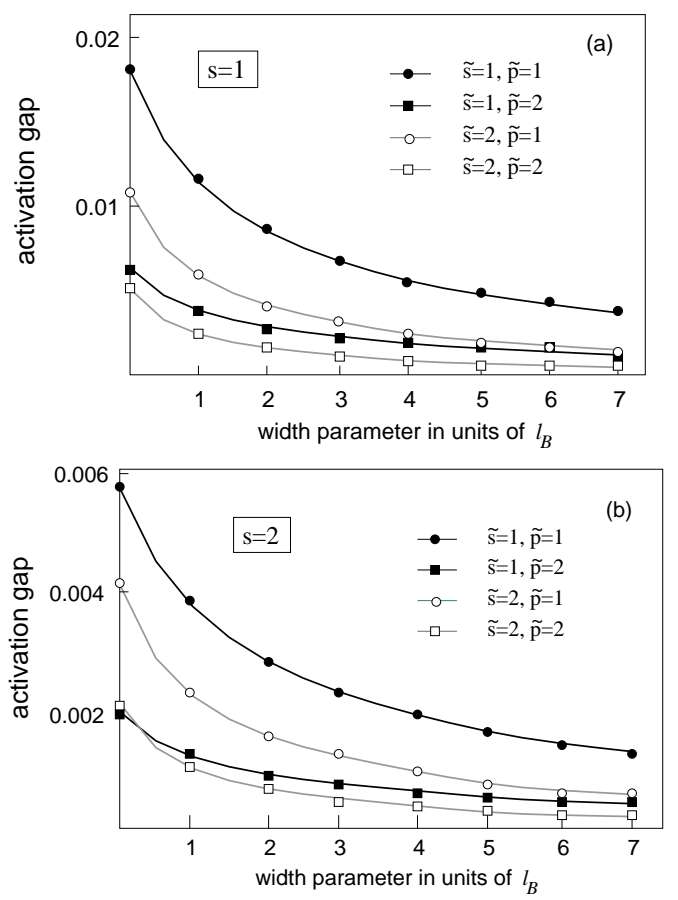

FIG. 3: Activation gaps. (a) $s=1: \nu=4 / 11$ (black circles), $\nu=6 / 17$ (white circles), $\nu=7 / 19$ (black squares), and $\nu=$ 11/31 (white squares); (b) $s=2: \nu=4 / 19$ (black circles), $\nu=6 / 29$ (white circles), $\nu=7 / 33$ (black squares), and $\nu=$ $11 / 53$ (white squares).

\section{Erratum}

A factor $n_{B}^{*}=1 / 2 \pi l_{B}^{* 2}$ is missing in Eq. (8). Its corrected version is

$$
\mathcal{F}_{n}^{p}(q)=n_{B}^{*} \sum_{n^{\prime}=p-n}^{p-1}\left|\left\langle n^{\prime}+n\left|\bar{\rho}^{p}(\mathbf{q})\right| n^{\prime}\right\rangle\right|^{2} .
$$

This error has led to an overestimation of the screening effect due to inter-CF-LL excitations. Consequently screening affects the values of the activation gaps less than originally thought. The corrected values for the gaps with $s=1$ and $p=1$

\begin{tabular}{|c||c|c|}
\hline$\tilde{s}=1$ & $\tilde{p}=1$ & $\tilde{p}=2$ \\
\hline$\nu^{*}$ & $1+1 / 3$ & $1+2 / 5$ \\
\hline$\nu$ & $4 / 11$ & $7 / 19$ \\
\hline \hline$\Delta^{a}$ & 0.018 & 0.0064 \\
\hline
\end{tabular}

\begin{tabular}{|c||c|c|}
\hline$\tilde{s}=2$ & $\tilde{p}=1$ & $\tilde{p}=2$ \\
\hline$\nu^{*}$ & $1+1 / 5$ & $1+2 / 9$ \\
\hline$\nu$ & $6 / 17$ & $11 / 31$ \\
\hline \hline$\Delta^{a}$ & 0.011 & 0.0052 \\
\hline
\end{tabular}

and for $s=2$ and $p=1$

\begin{tabular}{|c||c|c|}
\hline$\tilde{s}=1$ & $\tilde{p}=1$ & $\tilde{p}=2$ \\
\hline$\nu^{*}$ & $1+1 / 3$ & $1+2 / 5$ \\
\hline$\nu$ & $4 / 19$ & $7 / 33$ \\
\hline \hline$\Delta^{a}$ & 0.0057 & 0.0020 \\
\hline
\end{tabular}

\begin{tabular}{|c||c|c|}
\hline$\tilde{s}=2$ & $\tilde{p}=1$ & $\tilde{p}=2$ \\
\hline$\nu^{*}$ & $1+1 / 5$ & $1+2 / 9$ \\
\hline$\nu$ & $6 / 29$ & $11 / 53$ \\
\hline \hline$\Delta^{a}$ & 0.0041 & 0.0021 \\
\hline
\end{tabular}

in units of $e^{2} / \epsilon l_{B}$.

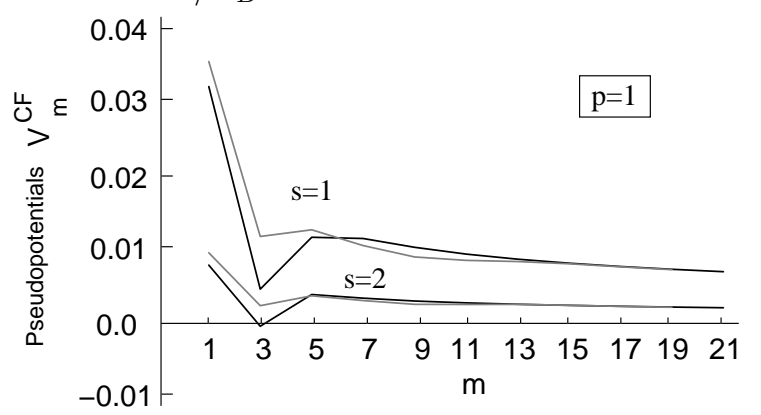

FIG. 4: Haldane's pseudopotentials for CF interaction both without screening (black line) and with screening (gray line); the lines are a guide to the eyes.

The corrected figures may be found in Figs. 3 and 4 The theoretical estimate for the activation gap at $\nu=$ $4 / 11$ for a width parameter $\lambda \simeq 6.3 l_{B}$ is $\Delta^{a}(s=1 ; \tilde{s}=$ $\tilde{p}=1) \simeq 0.004 e^{2} / \epsilon l_{B}$, which is on the order of $600 \mathrm{mK}$. The characteristic minimum of $V_{3}^{C F}$ persists even in the presence of screening (Fig. (4). 